\title{
EERS: Energy Efficient Rate Selection Approach for Wireless Battery Operated Networks
}

\author{
Liansheng $\mathrm{Tan}^{1}$, Chunyan $\mathrm{Su}^{2}$, and ${\mathrm{Jie} \mathrm{Li}^{3}}^{3}$
}

\begin{abstract}
For mobile ad hoc networks (MANETs) and sensor networks, due to the battery recovery effect, a node with longer idle period after finishing a transmission task will recover more over-consumed energy; however, longer recovery period requires shorter transmission period and hence higher transmission rate which leads to more energy consumption for transmission. Therefore, there is a tradeoff between the energy consumption for transmission and the energy recovery when minimizing the overall energy depletion at each node. This paper firstly defines a new metric termed actual energy depletion (AED), which quantifies the actual energy consumption during a transmission and recovery time unit of a node. We then describe the rate selection problem into an optimizing model to minimize AED subject to the channel's capacity constrain. We propose a novel energy efficient rate selection (EERS) approach for wireless battery operated networks. We analyze the mathematical procedure on how to obtain the required optimal rate for the proposed model and provide its implementation method in real networks in a distributive way. Numerical results are provided to show the efficiency of EERS in prolonging network lifetime.
\end{abstract}

\section{INTRODUCTION}

Mobile ad hoc networks (MANETs) and sensor networks are both energy constrained [1], [2] due to the fact that their nodes are mostly operated on batteries. Therefore, conserving battery power and prolong the network lifetime come to be critical issues, and subsequently designing energy efficient protocols for such networks also becomes an active research area.

The present work discusses rate selection schemes for energy constrained networks, and is motivated by the following observation: for a transmission task with bounded latency constraint, due to the battery recovery effect, a node with longer idle period after finishing a transmission task will recover more over-consumed energy; however, longer recovery period requires shorter transmission period and hence higher transmission rate which leads to more energy consumption for transmission. Therefore, there is a tradeoff between the energy consumption for transmission and the

\footnotetext{
1 Liansheng Tan is with Research School of Information Sciences and Engineering, The Australian National University, Canberra ACT 0200, Australia (Email: liansheng.tan@rsise.anu.edu.au).

${ }^{2}$ Chunyan $\mathrm{Su}$ is with the Computer Science Department of Central China Normal University, Wuhan 430079, PR. China (chunyan.su@gmail.com)

$3 \mathrm{Jie} \mathrm{Li}$ is with Graduate School of Systems and Information Engineering, University of Tsukuba, Tsukuba Science City, Japan (Email: lijie@cs.tsukuba.ac.jp).

Liansheng Tan's research is supported partially by National Natural Science Foundation of China under Grant No. 60473085, the Program NCET-05-0673 from Chinese Ministry of Education and partially by the Australian Research Council. Jie Li's research is is partially supported by Grand - in - Aid for Scientific Research (C No.16500064) of Japan Society the Promotion of Science (JSPS).
}

energy recovery when minimizing the overall energy depletion at each node.

Battery model has emerged as an important issue in designing energy efficient protocols for battery operated networks. Recent studies [3], [4], [5], [6] in battery technology reveal that the behavior of the battery discharging is nonlinear. Batteries tend to over consume power during discharging. However, the over-consumed power can be reimbursed [5], [6], [7] if the battery has sufficient idle time for recovery. That is, once the battery disconnected from load, it will try to recover and pick up some over-consumed power. So, if we guarantee that whenever the battery has discharged for a task load, there is always an idle period followed up, and then the battery can take recovery in time, and make it come back to a good state. However, the existing relevant rate selection schemes [8], [9], [10], [11] for energy constrained networks do not take the battery recovery effect into account, and hence fail to address the issue of trading off between the energy consumption for transmission and the energy recovery properly. Our present work considers this tradeoff in designing rate selection scheme. We suggest to select an optimal transmission rate at each node, according to the network current environment and batteries discharging profiles.

To quantify the actual energy consumption during a transmission, we firstly define a new metric termed Actual Energy Depletion (AED). AED is the balance between consumed energy for transmission and recovered energy during the followed idle period. Then we describe the rate selection problem into an optimizing model to minimize AED subject to the link's capacity constrain. Finally, we propose a novel energy efficient rate selection (EERS) approach for wireless energy constrained networks with the aim to decrease the overall energy depletion and prolong network lifetime. We analyze the mathematical procedure on how to obtain the required optimal rate at each node and provide its implementation method in real networks in a distributed way.

\section{RELATED WORKS}

IEEE 802.11 standard is specially designed for wireless networks. This standard mainly provides three different physical layer transmission protocols (802.11a, 802.11b, and $802.11 \mathrm{~g}$ ), and they all support multiple data rates at the physical layer. For example, for IEEE 802.11a, the possible data rates are $6,9,12,18 \ldots$ and $54 \mathrm{Mbps}$ and for IEEE $802.11 \mathrm{~b}$ the set of possible data rates is 1, 2, 5.5 and $11 \mathrm{Mbps}$. However, the IEEE 802.11 standard does not specify how to select or adapt the transmission rate dynamically under different network conditions.

For wireless networks, several rate selection mechanisms at the medium access layer have been proposed to utilize the multi-rate capability by automatically selecting the 
transmission rate according to the channel conditions. The Auto Rate Fallback (ARF) protocol [9] is the first commercially available one. With ARF, the sender will increase the data rate after consecutive successful transmission and reduce rate after failure. The Receiver Based Auto Rate (RBAR) protocol [10] lets the receiver choose the data rate based on the Signal to Noise Ratio (SNR) information. This method can adapt to channel conditions more quickly, but requires modifications to the IEEE 802.11 standard. The Opportunistic Auto Rate (OAR) protocol [11] opportunistically uses the high quality channel whenever it is available to send data packets. OAR depends on two mechanisms: it uses RBAR to access the media and uses the IEEE 802.11 mandated fragmentation field to hold the channel for an extended number of packet transmissions. However, the above three typical auto rate selection mechanisms have not considered conserving energy. The works [12], [13], [14], [15], [16] take the saving energy into account. Actually, transmission rate have great effect on energy diffusion. The DRS (Dynamic Rate-Selection) [8] has taken energy conserving into consideration. In DRS, the transmission rate is based on the available energy budget at each node or link, and nodes with large energy budgets transmit with higher data rates than small energy budget nodes, thus saving the energy of low battery level nodes. The energy-delay tradeoff is considered by bounding the total delay on a packet obeying a QoS constraint.

Once we consider the energy diffusion in energy constrained networks, one is forced to account for the battery discharging features. The main contribution of our present work is to consider battery discharging profile, and integrate the battery recovery effect into the rate selecting scheme.

\section{BASIC ASSUMPTIONS}

For the sake of simplicity, in order to obtain the optimal transmission rate at each node mathematically from an optimized problem, we need make some assumptions on the node state, traffic arriving process and network topology. These assumptions are reasonable.

\section{A. Network Topology}

Let us consider the sparse network topology as shown in Fig. 1. In [8], the authors described such a network as the one consisting of nodes that are set apart from each other and each node can only reach its direct neighbors. This topology constraint is set to extract the sole effect of varying the data rates on the network lifetime without being affected by other factors such as messages collision and interference from other nodes. For example, we consider a single connection flow A-B-C-D-E (shown in bold) in Fig. 1, while, when it is transmitting data, the total interference from other flows is neglectable due to the sparse network topology.

\section{B. Traffic Arriving Process}

For each node, it has some transmission tasks which arrival randomly. Each transmission task may contain a large number of packets and when a node has finished the transmission for all these packets, we call that this node has finished this transmission task. We assume the transmission tasks arrival process is Poisson Process, and the mean arrival rate is $\lambda$. Therefore, the intervals between two adjacent tasks obey exponential distribution with parameter $\lambda$. On average, there are $1 / \lambda$ time units between two arrivals. For transmission task without specified urgency level, we assume to finish it during $1 / \lambda$ time units. In other words, the average latency for a node's transmission tasks is bound to $1 / \lambda$.

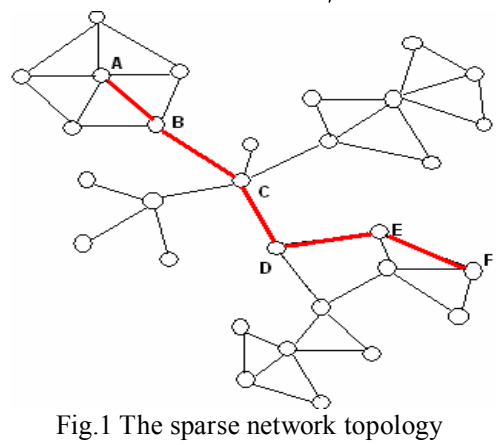

C. Node State

A node's states can be divided into three: active, idle and dead. During the active state, nodes can transmit, receive, or forward data to other nodes, and at the same time, its battery energy drains away; during the idle state, nodes are assumed to consume a negligible amount of energy due to its battery recovery effect; and finally when a node drains up all energy from its battery, it will die and can never do any task. Moreover, any existing route or path, which includes this dead node, will be invalid, and cannot transmit data any more.

Each node may have two roles, as the source node or as the destination node. For each <source, destination> pair, the optimal transmission rate is determined by the source node.

\section{EERS: An Energy Efficient Rate Selection Approach}

\section{A. Rate Selection Model}

To take the battery recovery effect into account, we define a new metric termed Actual Energy Depletion (AED) to quantify the actual energy consumption. AED is the balance that the consumed energy for transmission minus the recovered energy. Note that, in this rate selection model, we only consider energy consumption by transmitting power diffusion but our model can be easily adapted to include the receiving power diffusion.

\section{Actual Energy Depletion (AED):}

$$
E(x)=E_{c(i, j)} \cdot t_{c(i, j)}-E_{r(i, j)} \cdot t_{r(i, j)},
$$

where $E(x)$ is the Actual Energy Depletion at Node $i$ when allocate rate with $x$, which consists two parts, the energy consumed during transmission to Node $\mathrm{j}$, and the energy recovered in the idle time slots. $E_{c(i, j)}$ is the energy consumption for data transmission form Node $i$ to Node $j$ during one time slot; $t_{c(i, j)}$ is the mean time slots needed for completing the transmission task; $E_{r(i, j)}$ is energy recovered during one idle time slot; and $t_{r(i, j)}$ is the mean time period for recovery measured in time slot. Note that, the energy recovery occurs only during idle time slots. 
As mentioned above, we assume the transmission task obeys Poisson Arrival Process, and the average arrival rate is $\lambda$. Then, the total average time for transmission and recovery is the mean interval of task arrival, that is $t_{c(i, j)}+t_{r(i, j)}=1 / \lambda$. Moreover, $t_{c(i, j)}$ can be described as $M / x$, where $M$ is total amount packets for one transmission task and $x$ is the allocated transmission rate. Therefore, $t_{r(i, j)}$ can be expressed as $1 / \lambda-M / x$. However, if the traffic arriving process just follows a general distribution and then we can use the mean values for the parameters $t_{c(i, j)}$ and $t_{r(i, j)}$. We are now ready to describe the energy efficient rate selection problem into the following optimizing model to minimize AED subject to the channel's capacity constrain.

\section{Optimization Problem:}

$\min E(x)$

subject to $x \leq C$ over $\mathrm{x} \geq 0$, where $C$ is channel capacity. Note that, in (1), $E_{c(i, j)}$ depends on many factors, for instance, the wireless devices, the transfer distance, the transmission rate, the channel state, etc. Many work (e.g., [17]) assumed that the relationship between energy and data rate is directly proportional. In [17], the authors introduce a power consumption model: $p_{t}(i, k)=C_{i k} \cdot f_{i k}$, where $p_{t}(i, k)$ is the power dissipated at Node $i$ when transmitting to Node $k, f_{i k}$ is the transmission rate, ${ }^{C} i k$ is the power consumption cost of link (i, k). We can see that the author assumed the energy consumption for transmission is proportional to the transmission rate. However, more and more research [4], [5], [6], [8] reveals that the relationship between energy dissipation and transmission rate is nonlinear due to the power provided devices, such as batteries, have nonlinear discharging curve. Here, we propose a more accurate energy consumption model:

$$
E_{c(i, j)}=\left(\alpha+\beta \cdot d^{m}\right) \cdot f(x),
$$

where $f(x)$ is the energy cost function, and accounts for the ratio between the energy consumption $P$ to transmission rate $x$. That is, $f(x)$ equals to $P / x ; \alpha$ is a distance independent constant that accounts for the energy consumption by the digital processing and electronics $\beta$ is a constant dependent upon the antenna; $d$ is Euclidean distance between Node $i$ and Node $j ; m$ is the propagation loss coefficient.

Generally, transmission rate over a link may be influenced by the channel state, such as the noise and fading coefficients of channel, interference from other channels, etc. In [18], for a White Gaussian Noise Channel, the achievable rate could be approximated by the Signal to Interference ratio (SIR), given by

$$
x=\log \left(1+\frac{\mu \cdot P}{N+P_{I}}\right),
$$

where $x$ is transmission rate, $P$ is power required for successful transmission to the destination node, $\mu$ is the channel fading coefficient, $N$ is the channel noise, and $P_{I}$ is total interference from other nodes. Starting from (3), we are able to obtain the energy cost function $f(x)$ by the following mathematical deductions. The equation (3) is equivalent to

$$
e^{x}=1+\frac{\mu \cdot P}{N+P_{I}},
$$

that is

$$
\frac{P}{x}=\frac{N+P_{I}}{\mu} \cdot \frac{e^{x}-1}{x} .
$$

We are discussing the network consisting of nodes that are set apart from each other such that each node can only reach its direct neighbors. Therefore, the total interference from other nodes $P_{I}$ can be negligible. Based on the above mathematical manipulations we obtain the specific expression for $f(x)$ as following

$$
f(x)=\gamma \cdot \frac{e^{x}-1}{x},
$$

where we have denoted $\gamma=N / \mu$.

We assume that node's energy recovery occurs if and only if there are idle time slots for it. The recovery amount mainly depends on the recovery length and battery's chemical characteristic. For simplicity, we assume that battery recover one charge unit during one idle time slot, denoted as $U_{C}$. Then, the explicit expression for $E_{r(i, j)}$ is as

$$
E_{r(i, j)}=U_{C}
$$

Note that, $U_{C}$ depends on the battery type, and may vary from battery to battery. Here, we suggest to approximate $U_{C}$ with the product of battery rated discharge voltage $R_{V}$, and battery rated current $R_{I}$, which are usually recommended by the manufacturers, and explicitly labeled on the batteries. Specifically, for each battery, $U_{C}$ equals to $R_{v} \cdot R_{I}$. Sometimes, if the rated current is not labeled on the battery, we can indirectly obtain its value from another important parameter of battery: Theoretical Capacity, since they have the same numerical value.

On the basis of the above analyses, we obtain a specific expression for $E(x)$ :

$$
E(x)=\left(\alpha+\beta \cdot d^{m}\right) \cdot r \frac{e^{x}-1}{x} \cdot \frac{M}{x}-\left(\frac{1}{\lambda}-\frac{M}{x}\right) \cdot U_{C} .
$$

Then, the Optimization Problem can also be described as

$$
\min M \cdot \gamma \cdot\left(\alpha+\beta \cdot d^{m}\right) \frac{e^{x}-1}{x^{2}}-\left(\frac{1}{\lambda}-\frac{M}{x}\right) \cdot U_{C},
$$

subject to $x \leq C$ over $\mathrm{x} \geq 0$.

Note that, for each node, when implementing our EERS scheme for selecting the optimal rate, all the parameters are assumed to be constant and can be pre-determined.

We observe that the objective optimization function in (9) is non-linear. By non-linear optimization theory, when the objective function at its minimum, its first order derivative, denoted as $E^{\prime}(x)$, should be equal to zero. Here, we firstly calculate $E^{\prime}(x)$ as

$$
E^{\prime}(x)=M \cdot\left(\gamma \cdot\left(\alpha+\beta \cdot d^{m}\right) \frac{x \cdot e^{x}-2 e^{x+2}}{x^{3}}-U_{C} \frac{1}{x^{2}}\right) .
$$

From $E^{\prime}(x)=0$ we then have the following equation: 


$$
x \cdot e^{x}-2 e^{x}-\frac{U_{C}}{M \cdot \gamma\left(\alpha+\beta \cdot d^{m}\right)} x+2=0
$$

By denoting $\omega=\frac{U_{C}}{M \cdot \gamma\left(\alpha+\beta \cdot d^{m}\right)}$, (11) can be simplified as: $x \cdot e^{x}-2 e^{x}-\omega \cdot x+2=0$.

Equation (12) is non-linear and can be solved via applying some Numerical method, such as Interval Newton Method.

\section{B. Rate selection algorithm}

On the basis of the above analyses, we propose an energy efficient rate selection algorithm. Firstly, construct the objective optimization function, according to the current network environment. Then, solve this optimization function via applying the Interval Newton Method, and obtain the solution set, denoted as $A$. Finally, choose one from $A$ as the optimal rate in order to minimize the proposed metric, AED. The whole process is described into the following 5 steps.

Step1: Get the node's battery type, obtain the rated discharge voltage $R_{V}$ and the rated discharging current $R_{I}$, and then calculate $R_{V} \cdot R_{I}$ for $U_{C}$.

Step 2: Get the current channel state information, obtain the channel noise coefficient $N$ and the channel fading coefficient $\mu$, then calculate $N / \mu$ for $\gamma$.

Step 3: Calculate $\frac{U_{C}}{M \cdot \gamma\left(\alpha+\beta \cdot d^{m}\right)}$, and set $\omega$ with this value.

Step 4: Call the Procedure Rate Selection, to get the solution set of (8), denoted as Set $A$.

Step 5: Choose the optimal rate $X_{\text {optim }} \in A$ that satisfies

$$
X_{\text {optim }} \in \arg \min _{x_{i} \in A} E\left(x_{i}\right)
$$

Note that, the transmission route is pre-known, and thus each node knows its next-hop node id and the distance between them. For a given task, the total number of packets to transmit $M$ is known. Moreover, we assume that parameters for node's antenna loss coefficient $\beta$, propagation loss coefficient $m$, and the electronic energy $\operatorname{cost} \alpha$ are all pre-determined.

\section{Discussion on the energy-delay tradeoff}

In energy-constrained networks, one is usually forced to consider energy-delay tradeoff [8]. In DRS scheme [8], energy-delay tradeoff is considered by bounding the total delay on a packet obeying a QoS constraint. As mentioned above, our model can bound the average transmission delay on $1 / \lambda$, which accounts for the interval of transmission tasks arrival. For any transmission task without specified latency or urgency level description, we assume that the node can finish it during $1 / \lambda$ time units. To address the issue of energy-delay tradeoff, we now show that the model described by (9) can be modified to allow users to dynamically specify the acceptable latency for each transmission task. For a given latency upper limit $T$, which is usually specified by user, $T$ can be divided into two parts, the actual transmission time $t_{c(i, j)}$, and the recovery time $t_{r(i, j)}$, that is, $t_{c(i, j)}+t_{r(i, j)}=T$. If the user specifies its latency upper limit $T$, the optimization problem in
(9) is described as:

$$
\begin{aligned}
& \min M \cdot \gamma\left(\alpha+\beta \cdot d^{m}\right) \frac{e^{x}-1}{x^{2}}-\left(T-\frac{M}{x}\right) \cdot U_{C}, \\
& \text { subject to } \quad x \leq C, \frac{M}{x} \leq T, \text { over } \mathrm{x} \geq 0 .
\end{aligned}
$$

This modified model will be more flexible and ready to apply in real networks than the model described by (9) in the sense that, the solution to this optimization problem leads to an optimal rate selection scheme that satisfies the user specified latency requirement. Once the user specifies its acceptable latency for each transmission task, following the similar rate selection algorithm given for the optimization problem (9), one is able to yield the desired optimal rate selection.

\section{Performance Evaluation}

\section{A. Numerical Examples}

We consider the flow A-B-C-D-E-F in Fig. 1. There are five links: A-B, B-C, C-D, D-E and E-F. Each link is a <source, destination> pair. Along this flow, we assume that there are 100 packets to transmit, where the length of each packet is 10 $\mathrm{Kb}$. For each <source, destination> pair, rate selection algorithm is implemented at the source node. The battery level of each node is randomly set as follows: Node A: $10080 \mathrm{~J}$; Node B: 6480 J; Node C: 12240 J; Node D: 8208 J; Node E: $5832 \mathrm{~J}$; Node F: $8100 \mathrm{~J}$. We choose the typical values for $\alpha$ and $\beta: \alpha$ is $50 \mathrm{~nJ} / \mathrm{b}, \quad \beta$ is $100 \mathrm{pJ} / \mathrm{b} / \mathrm{m}^{2}$, and we will hold the same setting for $\alpha$ and $\beta$ in the sequel unless otherwise specified. The channel state parameter $\gamma$, we simply set with 1 , due to the ideal network condition assumption. We approximate the battery's one charge unit during one idle slot as mentioned in section III. For instance, we choose to use AMOi F9 lithium battery while the recommended discharging voltage is $2.5 \mathrm{~V}$, and the Theoretical Capacity is $600 \mathrm{mAh}$, then we can approximate, during one idle slot (we assume time slot length is 1second.), recovery amount is $1.32 \mathrm{~J}$. If then, choose the distance between Node A and Node B to be $100 \mathrm{~m}$, the optimal rate for link A-B can be obtained as $6.0 \mathrm{Kbps}$ via applying our EERS approach. As such, we can obtain the optimal rate for other links as given in TABLE I which shows different optimal rate for different link, where $d$ is the distance between the link's source node and destination node, $R_{V}$ is the source node's battery rated voltage, $R_{I}$ is the source node's battery rated current, and $X^{*}$ (as in bold) is the optimal transmission rate calculated according to our EERS scheme.

TABle 1: The Optimal Rate For The Links

\begin{tabular}{|c|c|c|c|c|}
\hline & $d(\mathrm{~m})$ & $R_{V}(\mathrm{~V})$ & $R_{I}(\mathrm{~mA})$ & $X^{*}(\mathrm{Kbps})$ \\
\hline A-B & 100 & 3.5 & 800 & $\mathbf{6 . 0}$ \\
\hline B-C & 100 & 3.0 & 600 & $\mathbf{5 . 6}$ \\
\hline C-D & 150 & 4.0 & 850 & $\mathbf{6 . 2}$ \\
\hline D-E & 150 & 3.0 & 760 & $\mathbf{5 . 8}$ \\
\hline E-F & 100 & 2.7 & 600 & $\mathbf{5 . 5}$ \\
\hline
\end{tabular}

TABLE 2 shows the detailed energy dissipation for each link, where $T_{\text {trans }}$ is the required transmission time slots, $T_{\text {rec }}$ is the 
recovery time slots, $E_{\text {trans }}$ is the energy consumption for transmission, $E_{\text {recv }}$ is the recovered energy in $T_{\text {recv }}$ time slots, and $E$ (as in bold) is the total energy dissipated which equals to $E_{\text {trans }}-E_{\text {recv }}$.

TABLE 2: THE LINK LEVEL ENERGy DisSIPITION

\begin{tabular}{|c|c|c|c|c|c|}
\hline & $T_{\text {trans }}(\mathrm{s})$ & $E_{\text {trans }}(\mathrm{J})$ & $T_{\text {recv }}(\mathrm{s})$ & $E_{\text {recv }}(\mathrm{J})$ & $E(\mathrm{~J})$ \\
\hline A-B & 167 & 117.38 & 23 & 64.40 & $\mathbf{5 2 . 9 8}$ \\
\hline B-C & 179 & 90.21 & 11 & 19.80 & $\mathbf{7 0 . 4 1}$ \\
\hline C-D & 162 & 134.32 & 28 & 78.01 & $\mathbf{5 6 . 3 1}$ \\
\hline D-E & 172 & 102.78 & 18 & 39.01 & $\mathbf{6 3 . 7 7}$ \\
\hline E-F & 182 & 84.58 & 8 & 12.96 & $\mathbf{7 1 . 6 2}$ \\
\hline
\end{tabular}

Now, we come to see the relationship between the transmission rate and the energy consumption. We take A-B link for example. We choose different values for transmission rate: $5.0,5.3,5.5,6.0,6.5,6.7$ and 7.0 , and respectively compute the energy dissipation. The comparison results are shown in TABLE 3 and Fig 2. We can see from both TABLE 3 and Fig 2 that, $6.0 \mathrm{Kbps}$ is the optimal rate for A-B link.

TABLE 3: ENERGy DisSiPATION ON LiNK A-B OVER DifFERENT RATES

\begin{tabular}{|c|c|c|c|}
\hline$X^{*}(\mathrm{Kbps})$ & $E_{\text {trans }}(\mathrm{J})$ & $E_{\text {recv }}(\mathrm{J})$ & $E_{(\mathrm{J})}$ \\
\hline 5.0 & 61.91 & 0 & 61.91 \\
\hline 5.3 & 74.51 & 3.69 & 70.82 \\
\hline 5.5 & 84.55 & 22.95 & 61.60 \\
\hline $\mathbf{6 . 0}$ & $\mathbf{1 1 7 . 3}$ & $\mathbf{6 4 . 4 0}$ & $\mathbf{5 2 . 9 8}$ \\
\hline 6.5 & 165.05 & 101.23 & 63.82 \\
\hline 6.7 & 203.65 & 120.23 & 83.42 \\
\hline 7.0 & $234.78 \mathrm{~J}$ & $132.0 \mathrm{~J}$ & $102.78 \mathrm{~J}$ \\
\hline
\end{tabular}

The energy consumption in our EERS scheme is compared against the ARF mechanism where rates are selected randomly, the no energy efficient management base case (we call it NO-EM) mechanism where all the nodes transmit with a default maximum rate of $8 \mathrm{Mbps}$, and the DSR scheme. TABLE 4 and Fig 3 display the comparison results. We can see from TABLE 4, Fig. 3 that, EERS scheme saves more energy than the other three schemes.

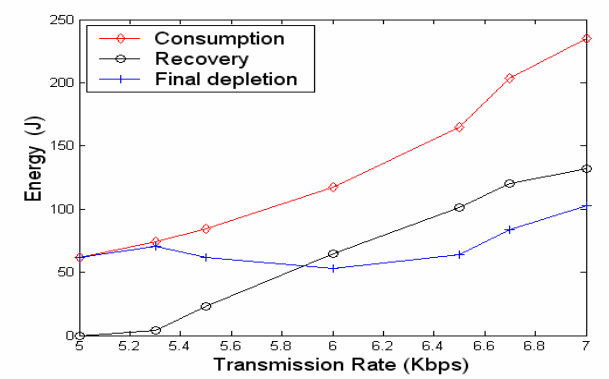

Fig 2 Energy dissipation over different rates on Link A-B

TABLE 4: ENERGy DisSiPATION (EERS, DRS, ARF, NO-EM)

\begin{tabular}{|c|c|c|c|c|}
\hline & EERS (J) & DRS (J) & ARF (J) & NO-EM (J) \\
\hline A-B & 52.98 & 80.56 & 84.42 & 98.20 \\
\hline B-C & 70.41 & 105.28 & 100.32 & 114.06 \\
\hline C-D & 36.31 & 58.76 & 66.24 & 76.26 \\
\hline D-E & 63.77 & 89.92 & 92.02 & 105.281 \\
\hline E-F & 71.62 & 112.56 & 109.56 & 121.27 \\
\hline
\end{tabular}

\section{B. Simulation Investigation}

\section{1) Simulation Setup}

We focus on two objectives to evaluate the performance gains from EERS: (1) network lifetime; (2) network average residual energy. All the results presented here are based on $n s 2.28$, and the simulation scenario is similar to [8]. We choose the sparse network topology, as shown in Fig. 6, and each node can only be reachable by its immediate neighbors. 50 nodes are distributed within a $500 \times \leftarrow 500$ unit-square region, and each

flow of UDP traffic is generated from an MPEG trace file. There are four flows: flow 1 (A-F), flow 2 (H-L), flow 3 (M-P) and flows $4(\mathrm{R}-\mathrm{W})$. For each link, the transmission rate is determined by the source node. The duration of each time slot is 1 sec. All network control packets such as request-to-send, clear-to-send, and route discovery are transmitted under the minimum bit-rate (we choose $2 \mathrm{Mbps}$, here).To compare the performance gains with DRS [8], all packets are routed using Dynamic Source Routing (DSR), the same as in [8]. Note that, EERS is a general approach, and can be easily adapted to other routing protocols.
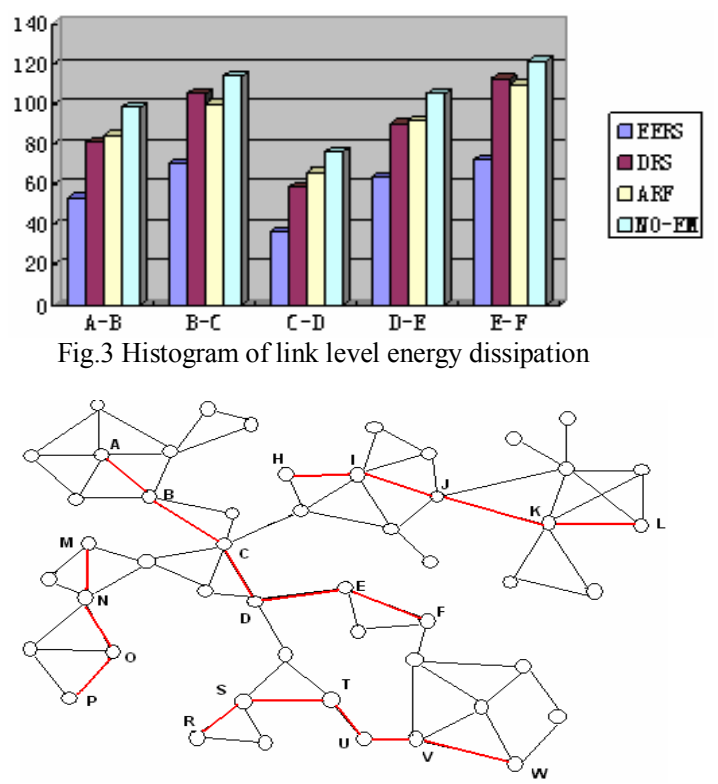

Fig.6 Simulation network topology

\section{2) Lifetime Extension Evaluation}

In our simulation lifetime is measured in time slots as the duration of time until the first node failure [8] due to battery exhaustion. Due to the sparse network topology, this measurement is practical in the sense that a single node may be crucial to the topology maintenance of the whole network.

The network lifetime under our EERS scheme is compared against that under DSR, ARF, and NO-EM mechanisms. We use the topology as shown in Fig. 6 while varying the battery level at each node. Here, we take a close look into the energy level variation process of each flow. Firstly, we define a flow's energy level to be the sum of residual energy of all nodes along this flow. The energy level variation of each flow during a transmission is shown in Fig. 5 to Fig. 8. We can see from them, there are several inflexions in each figure, which mean the node's battery switch to recovery phase, and the energy level increase during these recovery process due to some 
over-consumed energy been picked up.

Fig. 9 and Fig. 10 both illustrate the relationship between network lifetime and network initial energy level. We compare the lifetime performance under EERS scheme with DRS, ARF, and NO-EM. Lifetime is measured in time slots until the first node failure.
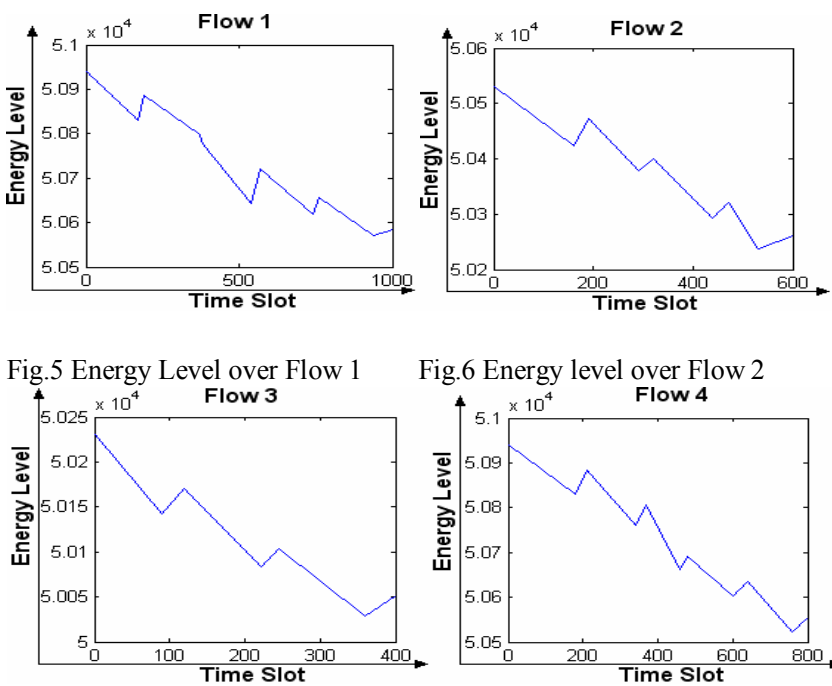

Fig.6 Energy level over Flow 2

Fig.7 Energy Level over Flow 3

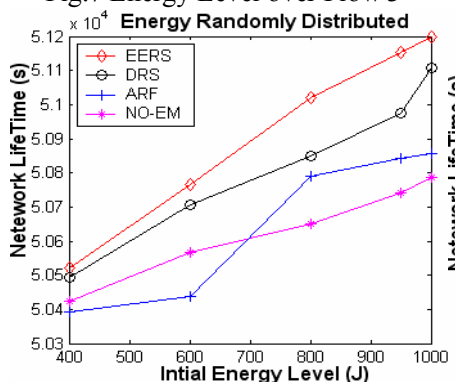

(a)

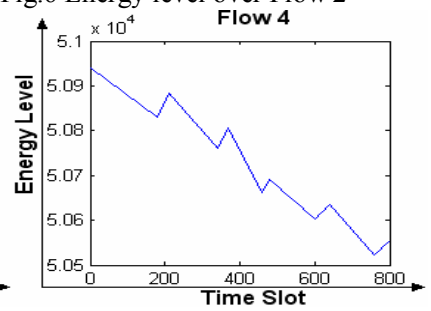

Fig.8 Energy Level over Flow 4

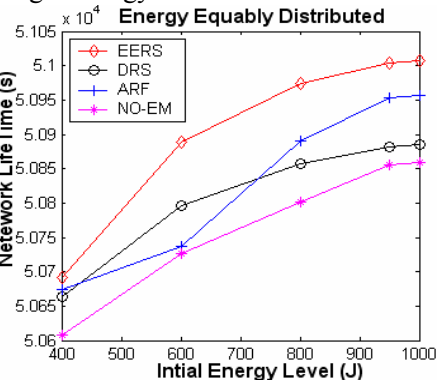

(b)
Fig.9 Network Lifetime for EERS, DRS, ARF, NO-EM

Figs. 9(a) and 9(b) display the network lifetime under the proposed rate selection scheme EERS and that under DRS, ARF and NO-EM, respective for the two cases: (1) the initial energy is randomly distributed at each node; and (2) the initial energy is equally distributed at each node. The network lifetime is significantly prolonged under the proposed rate selection scheme in both cases. Specifically, EERS scheme makes network lifetime extension reach as high as $20 \%$ longer than DRS, $35 \%$ longer than the NO-EM base case with no power-management. Moreover, we find that ARF does not always outperform NO-EM (occasionally, it can outperform DRS) due to its randomly rate selection policy. The whole simulations run 15 times for each network configuration and the confidential interval for all data points at confidential level $90 \%$ is approximately within 5 to 15 percent on the average.

\section{CONCLUSION}

In this paper, we propose a new energy efficient rate selection scheme, named EERS, for extending the lifetime of battery operated networks. The aim is conserving energy, especially to pick up the over-consumed power, via selecting the optimal transmission rate and well scheduling the battery state to guarantee the battery's recovery time. EERS can be implemented in a distributed way. The numerical results show that, for a network if selecting transmission rates at each source node according to EERS approach outlives that selecting transmission rates according to DRS, ARF or NO-EM. As a result, a data path using variable rate outlives a path with constant data rates. EERS scheme makes network lifetime extension reaches to $20 \%$ longer than DRS, $35 \%$ longer than the NO-EM base case with no power-management.

\section{REFERENCES}

[1] B. M. Sadler, "Fundamentals of energy-constrained sensor network systems", IEEE Aerospace and Electronic Systems Magazine, vol. 20, pp. 17-35, August 2005.

[2] M. Wiczanowski, Y. Chen, S. Stanczak, and H. Boche, "Optimal energy control in energy-constrained wireless networks with random arrivals under stability constraints," in Proceedings of IEEE Workshop on Signal Processing Advances in Wireless Communications, New York, 2005, pp. 831-836.

[3] C. F. Chiasserini and R. R. Rao, "Energy efficient battery management," IEEE Journal on Selected Areas in Communications, vol. 19, no.7, pp. 1235-45, July 2001.

[4] D. Panigrahi et al., "Battery life time estimation of mobile embedded systems," in Proceedings of the 14th International Conference on VLSI Design, Bangalore, 2001, pp. 57-63.

[5] R. Rao, S. Vrudbula and D. N. Rakbmatov, "Battery modeling for energy-aware system design," IEEE Computer, vol. 36, pp. 77-87, December 2003.

[6] D. Rakhmatov and S. Vrudhula, "Energy management for battery-powered embedded systems," ACM Transactions on Embedded Computing Systems, vol. 2, no. 3, pp. 277-324, August 2003.

[7] Y. Yang and C. Ma, "Battery-aware routing in wireless ad hoc networks - part I: energy model," in Proceedings. of the 19th International Teletraffic Congress, Beijing, 2005, pp. 293-302.

[8] N. AbouGhazaleh, P. Lanigan, S. Gobriel, D. Mosse, and R. Melhem, "Dynamic rate-selection for extending the lifetime of energy-constrained networks," in Proceedings of the 23rd IEEE International Performance, Computing and Communications Conference, Phoenix, 2004, pp. 553-558.

[9] A. Kamerman and L. Monteban, "WaveLAN II: A high-performance wireless LAN for unlicensed band," Bell Labs Technical Journal, vol. 2, no. 3, pp. 118-133, Summer 1997.

[10] G. Holland, N. Vaidya, and P. Bahl, "A rate-adaptive MAC protocol for multi-hop wireless networks," in Proceedings of ACM MOBICOM'01, Rome, 2001, pp. 236-251.

[11] B. Sadeghi, V. Kanodia, A. Sabharwal, and E. Knightly, "Opportunistic media access for multirate Ad Hoc networks,", in Proceedings of ACM MobiCom, Atlanta, 2002, pp. 24-35.

[12] R. Min, M.Bhardwaj, S.Cho, E.Shih, A.Sinha, A.Wang, and A. Chandrakasan, "Low-Power wireless sensor networks," in Proceedings of the 14th International Conference on VLSI Design, Bangalore, India, 2001, pp. 205-210.

[13] V. Raghunathan, C. Schurgers, S. Park, and M. Srivastava, "Energy aware wireless micro sensor networks," IEEE Signal Processing Magazine, vol. 19, no. 2, pp. 40-50, March 2002.

[14] L. Shang, L. Peh, and N. Jha, "Dynamic voltage scaling with links for power optimization of interconnection networks", in Proceedings of the 9th International Symposium on HPCA, Anaheim, 2003, pp. 79-90.

[15] A. Sinha, and A. Chandrakasan, "Operating system and algorithmic techniques for energy scalable wireless sensor networks," in Proceedings of the 2nd International Conference on Mobile Data Management, Hong Kong, 2001, pp. 199-209.

[16] G. Wei, J. Kim, D. Liu, S. Sidiropoulos, and M. Horowitz, "A variable-frequency parallel I/O interface with adaptive power-supply regulation," IEEE Journal of Solid-State Circuits, vol. 35, no. 11, pp. 1600-1610, November 2000.

[17] Y. T. Hou, Y. Shi, and H. D. Sherali, "Rate allocation in wireless sensor networks with network lifetime requirement," in Proceedings of the 5th ACM MobiHoc' 04, Tokyo, 2004, pp. 67-77.

[18] M. Bhardwaj and A. Chandrakasan, "Bounding the lifetime of sensor networks via optimal role assignments," in Proceedings of INFOCOM 2002, New York, 2002, pp. 1587-1596. 